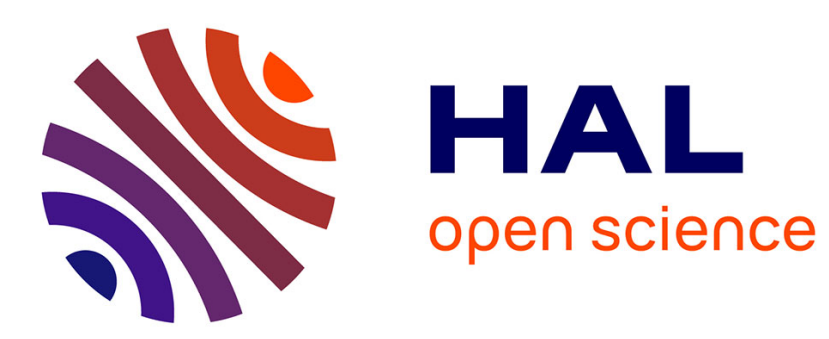

\title{
Contactless viscosity measurement by oscillations of gas-levitated drops
}

\author{
M Perez, L Salvo, M Suéry, Y Bréchet, M Papoular
}

\section{To cite this version:}

M Perez, L Salvo, M Suéry, Y Bréchet, M Papoular. Contactless viscosity measurement by oscillations of gas-levitated drops. Physical Review E , 2000, 61 (3), pp.2669-2675. 10.1103/PhysRevE.61.2669 . hal-01524864

\section{HAL Id: hal-01524864 \\ https://hal.science/hal-01524864}

Submitted on 16 Jun 2017

HAL is a multi-disciplinary open access archive for the deposit and dissemination of scientific research documents, whether they are published or not. The documents may come from teaching and research institutions in France or abroad, or from public or private research centers.
L'archive ouverte pluridisciplinaire $\mathbf{H A L}$, est destinée au dépôt et à la diffusion de documents scientifiques de niveau recherche, publiés ou non, émanant des établissements d'enseignement et de recherche français ou étrangers, des laboratoires publics ou privés. 


\title{
Contactless viscosity measurement by oscillations of gas-levitated drops
}

\author{
M. Perez, * L. Salvo, and M. Suéry \\ Génie Physique et Mécanique des Matériaux, ESA CNRS No. 5010, Boîte Postale 46, 38402 Saint Martin d'Hères Cedex, France \\ Y. Bréchet \\ Laboratoire de Thermodynamique et Physico-Chimie Métallurgiques, UMR CNRS No. 5614, Boîte Postale 75, \\ 38402 Saint Martin d'Hères Cedex, France \\ M. Papoular \\ Centre de Recherche sur les Très Basses Températures, CNRS, Boîte Postale 166, 38042 Grenoble Cedex, France
}

(Received 28 May 1999)

\begin{abstract}
A recently demonstrated aerodynamic levitation technique is used to perform contactless viscosity measurements. Classical models dealing with free oscillations of droplets without gravity cannot correctly describe the correspondence between damping coefficient and viscosity. An energetic approach taking into account the shape of the drop, and the velocity field of the liquid inside the drop, is introduced, leading to good agreement between experimental measurements and known viscosities of glycerol-water mixtures. Nonlinear effects are also investigated and experimental results are compared with the theory of anharmonic oscillations.

PACS number(s): 47.27.Wg, 47.55.Dz, 47.20.Gv, 47.80.+v
\end{abstract}

\section{INTRODUCTION}

The gas-levitated droplet technique has been developed in order to perform contactless processing and to improve the homogeneity of glass [1,2]. More recently, it has been exploited to perform contactless measurement of physical properties of liquids (such as surface tension and viscosity [3-5]). To study the solidification process of alloys, the absence of contact, which is a major source of heterogeneous nucleation, allows in situ measurements of bulk viscosity and surface tension.

Compared with the magnetic levitation technique, which has been used to perform contactless measurement of surface tension [6,7], viscosity in microgravity [8], density, and enthalpy [9], the gas-film-levitation technique is suitable for insulating liquid materials. Moreover, dissipation measurements, even in a liquid metal drop, will hit the viscosity parameter directly instead of a combination of viscosity and Joule dissipation due to eddy currents [8]. In order to measure surface energy and viscosity of liquids, one can study droplet oscillations. The response of the droplet triggered by a given frequency exhibits a resonance. In earth's gravitational field, the position of the resonance peak is governed by surface energy (restoring force) and density (inertial effect), whereas the width of this peak is related to the dissipative terms, namely, the viscosity of the liquid. In this paper, we focus our attention on the determination of viscosity of an oscillating droplet in an external gravitational field. We stress the fact that what we are discussing here is the deformation amplification of our droplet under vibrational excitation of the setup. As mentioned in our previous paper [10], this is evocative of a Faraday instability.

Lamb [11] (see also Ref. [12]) gave the resonance mode frequencies $f_{r}$ and resonance peak width $\Delta f_{r}$ for a free os-

*Electronic address: Michel.Perez@gpm2.inpg.fr cillating drop of volume $V$, radius $R$, surface energy $\sigma$, density $\rho$, and viscosity $\mu$, in the absence of gravity. For the mode $\ell$, he obtained

$$
\begin{aligned}
& f_{r}=\sqrt{\ell(\ell-1)(\ell+2) \sigma /(3 \rho \pi V)}, \\
& \Delta f_{r}=(\ell-1)(2 \ell+1) \mu /\left(2 \pi \rho R^{2}\right) .
\end{aligned}
$$

Very few systematic experiments exist on the variation of the oscillating modes under gravity with respect to volume or nature of the liquid $[3,4]$. With the magnetic levitation technique, the effects of the magnetic and gravitational fields on the oscillation frequencies have been calculated [13] allowing an accurate surface tension measurement [6,7]. However, no explanation has been proposed for the effect of the gravitational field on the width of the resonance peak. In the present paper, the effect of gravity and gas flux on the equilibrium shape is evaluated. The influence of the droplet shape on the resonance frequency is investigated, taking into account the real shape of the droplet instead of the ellipsoidal approximation used in Ref. [4]. An energetic approach leads to the interpretation of the viscous dissipation. For large excitation amplitude, nonlinear effects, theoretically predicted in Ref. [10], are also investigated. Systematic experiments with droplets of varying volume and viscosity are performed and the results are compared with these simple analytical models.

\section{EXPERIMENTAL SETUP AND MATERIALS}

The apparatus is schematically depicted in Fig. 1, and described in detail in Ref. [4]. A liquid droplet stands on a gas layer coming through a pressurized porous membrane (diffuser). Only the $\ell=2$ mode (oscillation between probate and oblate shapes) has been studied because of its relative high oscillation amplitude.

The drop is excited through an electromagnetic vibrator 


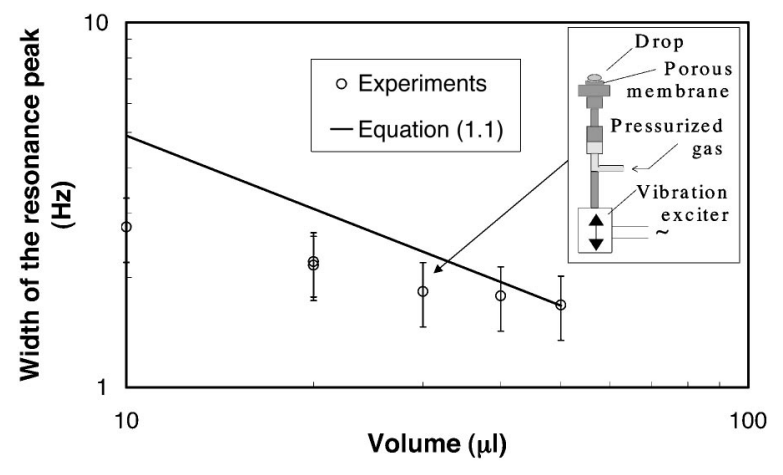

FIG. 1. Width of the resonance peak of a $30 \%$ glycerol droplet as a function of the volume: experiments (dots) and theory from Eq. (1.1) (line).

providing vertical oscillation to the system (diffuser-drop). Frequency and amplitude of the excitation are adjustable in a wide range (from 1 to $100 \mathrm{~Hz}$ for the frequency and 0 to $100 \mu \mathrm{m}$ for the amplitude). A video system allows drop profile measurements with an accuracy of $10 \mu \mathrm{m}$. Scanning in frequency gives the resonance peak. Temperature is measured by means of a thermocouple inserted in the diffuser.

In this work, experimental measurements have been performed at room temperature $(298 \pm 1 \mathrm{~K})$ on drops of waterglycerol mixtures with various fractions of glycerol (from $20 \%$ to $85 \%$ ). The physical parameters for water and glycerol are listed in Table I. The volume of the drop varies from $V=10 \mu \mathrm{l}$ to $V=100 \mu \mathrm{l}$. Some experiments were performed on calibrated silicon oil $(\mu=10.1 \mathrm{mPas}, \rho$ $=900 \mathrm{~kg} / \mathrm{m}^{3}, \quad \sigma=0.017 \mathrm{~J} / \mathrm{m}^{2}$ at $\left.298 \mathrm{~K}\right)$ provided by Brookfield Ltd.

\section{EXPERIMENTAL RESULTS}

The width of the resonance peak was measured at half height. Its variation with the droplet volume is shown in Fig. 1. Equation (1.1) with no gravity effects is unable to describe the results. As demonstrated in [4], gravity effects on the drop shape have to be taken into account, because the radius of the droplet is close to the capillary length for waterglycerol $\left(l_{c}=\sqrt{\sigma / \rho g}=2.5 \mathrm{~mm}, g\right.$ being the gravity acceleration).

A typical resonance peak is plotted in Fig. 2 for two different amplitudes of excitation. The small amplitude peak is symmetrical and accurately fitted by a simple viscoelastic model. However, if the excitation amplitude is too large, the resonance peak starts to be distorted, leading to a hysteresis in the amplitude-frequency diagram. This phenomenon is characteristic of nonlinear effects associated with anharmonicity. These first results underline the need for theoretical approach better adapted to gas-film-levitated drop concerning (a) the nonspherical equilibrium shape, (b) the resonance

TABLE I. Physical properties of water and glycerol at $298 \mathrm{~K}$

\begin{tabular}{lccc}
\hline \hline & $\begin{array}{c}\text { Viscosity } \\
(\mathrm{mPa} \mathrm{s})\end{array}$ & $\begin{array}{c}\text { Surface energy } \\
\left(\mathrm{J} \mathrm{m}^{-2}\right)\end{array}$ & $\begin{array}{c}\text { Density } \\
\left(\mathrm{kg} \mathrm{m}^{-3}\right)\end{array}$ \\
\hline Water & 1 & 0.073 & 1000 \\
Glycerol & 1700 & 0.063 & 1273 \\
\hline \hline
\end{tabular}

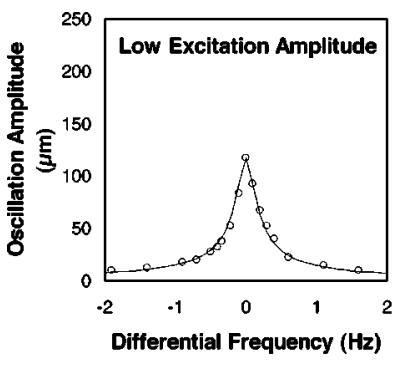

(a)

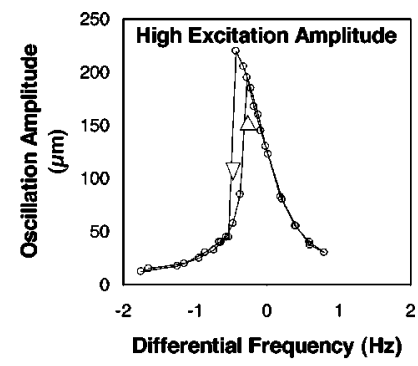

(b)
FIG. 2. Resonance peak of a gas-levitated droplet for low (a) and high (b) excitation amplitudes. Note the hysteretic behavior present in (b).

frequency, (c) the resonance peak width, and (d) the effect of excitation amplitude on the peak shape.

\section{EQUILIBRIUM SHAPE OF THE LEVITATED DROPLET}

\section{A. Laplace equation}

In order to understand the parameters influencing the equilibrium shape, a comparison is made between the theoretical shape of a sessile drop with a $180^{\circ}$ contact angle and photography of the real shape.

The shape of a sessile drop with $180^{\circ}$ contact angle depends only on the surface tension $\sigma$, the density $\rho$ of the liquid, and the gravity $g$. At each point of the surface, the Laplace equation has to be satisfied. Due to the revolution symmetry axis, the Laplace equation is equivalent to (see Ref. [14])

$$
\frac{d \theta}{d s}=-\frac{\sin \theta}{X}+\frac{\rho g}{\sigma} Z+\frac{2}{r_{c}} .
$$

$\theta$ is the angle defined in Fig. 3, $s$ is the curvilinear coordinate, $r_{c}$ is the radius of curvature at the top of the drop, $X$ is the horizontal coordinate, and $Z$ is the vertical coordinate. The profile of the sessile drop is calculated by solving Eq. (4.1) numerically at each point of the droplet surface and adjusting $r_{c}$ to obtain the correct volume.

In Fig. 4, it can be observed that the real shape of the levitated drop is almost exactly fitted by the shape of a $180^{\circ}$ contact angle sessile drop. The first consequence is that the equilibrium shape of the drop depends only on the ratio $\rho g / \sigma$ and is barely perturbed by the experimental conditions (gas flux, diffuser). The second consequence is the possibility of measuring the ratio $\rho g / \sigma$ and deducing the surface

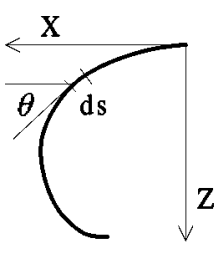

(a)

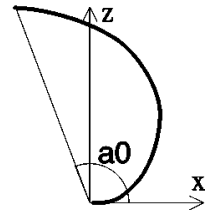

(b)
FIG. 3. Geometrical definition for the profile description (see Sec. IV): (a) profile solution of Laplace equation [Eq. (4.1)] and (b) profile described with the Archimedean arc [Eq. (4.2)]. 


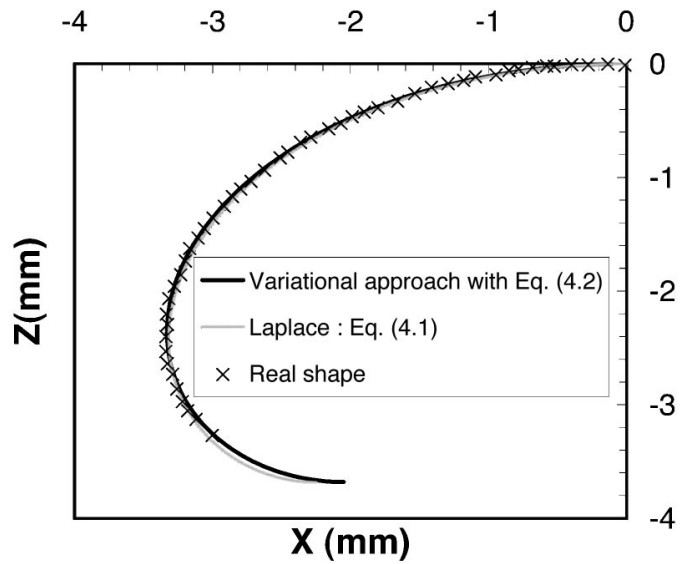

FIG. 4. Comparison between the experimental profile $(\times \times \times)$, the profile solution of Laplace equation (gray line), and the profile resulting from the variational approach using archimedian spiral (black line).

tension, just by fitting the calculated profile with the real profile, which is much more accurate than measuring a sessile drop contact angle.

\section{B. Variational approach with an Archimedean spiral arc}

Although it is difficult to find an analytical solution of Eq. (4.1), a numerical solution is not quite satisfactory. However, it seems that a flattened Archimedean arc could describe accurately the profile of the levitating drop. The equation of such a profile would be

$$
\begin{aligned}
& x(\alpha)=a \alpha \cos \alpha, \\
& z(\alpha)=b a \alpha \sin \alpha .
\end{aligned}
$$

Parameter $a$ describes the size and parameter $b$ the flattening of the shape. $\alpha$ varies from 0 to $\alpha_{0}$ (corresponding to the top of the drop where $d z / d x=0$ ) (see Fig. 3). If $a$ and $b$ are properly chosen, the Archimedean arc can fit the real profile. At that point, $a$ and $b$ have no physical meaning, which is not satisfactory. One would like to have an estimation of these parameters as a function of the drop properties $\sigma, \rho, g$, and $V$. The variational approach of Ref. [4] developed with an ellipsoidal profile can be used with the more realistic profile described by Eq. (4.2). The volume is given by

$$
V=\int_{0}^{\alpha_{0}}\left[x(\alpha)-x\left(\alpha_{0}\right)\right]^{2} \pi a b[\sin \alpha+\alpha \cos \alpha] d \alpha .
$$

$V$ is kept constant so that the droplet shape depends only on one parameter, chosen here to be $b$. The total energy of the system at rest can be calculated as the sum of gravitational potential and surface energy:

$$
E=\sigma S+\rho V g z_{m}
$$

$z_{m}$ is the droplet center of mass, which is the solution of the equation

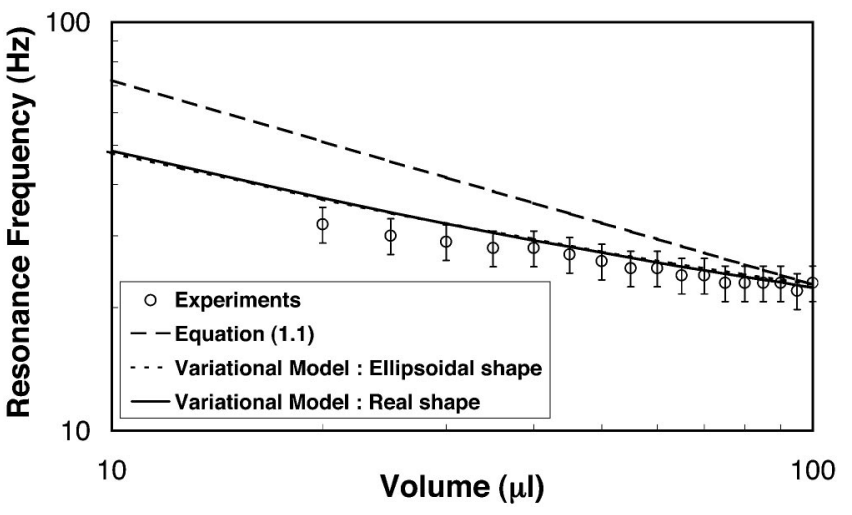

FIG. 5. Comparison of the experimental results for the resonance frequency with the estimation from variational approaches using ellipsoidal (- - ), or Archimedean shapes (-). The solution given by Eq. (1.1) is shown for comparison.

$$
\begin{aligned}
& \int_{0}^{\alpha_{0}}\left[z(\alpha)-z_{m}\right]\left[x(\alpha)-x\left(\alpha_{0}\right)\right]^{2} \pi a b[\sin \alpha+\alpha \cos \alpha] d \alpha \\
& \quad=0
\end{aligned}
$$

The energy minimum corresponds to a stable equilibrium position at $b=b_{e q}$. The same strongly asymmetric profile as in Ref. [4] is observed. For a given volume and a liquid of known density and surface tension, the value of $b_{e q}$ can be calculated, leading to the determination of the equilibrium droplet profile. Figure 4 compares the experimental profile, the exact profile derived from the Laplace equation, and the profile determined with the variational approach. The reasonably good agreement validates the variational approach.

\section{RESONANCE FREQUENCY APPROXIMATION: COMPARISON BETWEEN THE REAL AND THE ELLIPSOIDAL SHAPE}

Once the energetic profile is determined, the resonance frequency is given by the stiffness of the energy curve, i.e., the second derivative of $E$. In the case of an ellipsoidal shape [4], the derivation parameter is obvious: the center of gravity position. With such a geometry, this point corresponds to the center of symmetry. The surface forces and the volume forces can be assumed to be applied at that unique point. However, in the case of a nonsymmetric shape, such as the one induced by gravity, this reduction to a single resulting force applied at the center of gravity is no longer possible. Nevertheless, the resulting force is along the vertical axis. The dynamics of the system will be approximated by the dynamics of a specific point along the $z$ axis whose position between the two poles of the droplet will be chosen in order to get the closest fit between calculated and experimental frequencies. The aim of the present simplified model is to predict the influence of the volume on the characteristics of the resonance.

Figure 5 shows the resonance frequency as a function of the droplet volume. Assuming either an ellipsoidal shape or an approximation with Archimedean spirals leads to identical results since the two curves are close and parallel. On this basis, the droplet shape will be described as ellipsoidal in the remaining part of this paper. 


\section{VISCOSITY APPROXIMATION: AN ENERGETIC APPROACH}

The width of the resonance peak is associated with the dissipation processes occurring within the vibrating droplet. The dissipated power will be described first in a purely phenomenological manner using a viscoelastic model (A). A more direct interpretation will be given thanks to an approximate solution of the Navier-Stokes equation for the fluid flow in the droplet (B).

\section{A. The viscoelastic model}

For amplitude small enough to stay in the linear domain, the vibrating droplet can be modeled by the classical viscoelastic harmonic oscillator, whose motion is governed by

$$
\ddot{R}_{p}+2 \lambda \dot{R}_{p}+\omega_{0}^{2} R_{p}=\frac{F}{M} \cos \omega t .
$$

This gives

$$
\begin{gathered}
R_{p}=R_{p_{0}}+\Delta R_{p} \cos (\omega t+\varphi), \\
\Delta R_{p}=\frac{F}{2 M \omega_{0} \sqrt{\left(\omega-\omega_{0}\right)^{2}+\lambda^{2}}},
\end{gathered}
$$

where $R_{p}$ is the polar radius of the ellipsoid, $M$ the mass of the system, $\lambda=\pi \Delta f_{r}$ the dissipation coefficient, $\omega_{0}=2 \pi f_{r}$ the resonance angular frequency, $F$ the amplitude of the excitation force, $\omega$ the angular frequency of the excitation force, $R_{p_{0}}$ the polar radius at equilibrium introduced in Ref. [4], and $\Delta R_{p}$ the amplitude of the oscillations. This assumption is supported by the excellent correlation between an experimental resonance peak and Eq. (6.2) for small amplitudes of excitations (see Fig. 2).

The dissipated viscous power $P_{v}$ at the resonance frequency is given by (see Ref. [16])

$$
\left\langle P_{v}\right\rangle=M \lambda \omega_{0}^{2} \Delta R_{p}^{2}
$$

\section{B. The velocity field into the oscillating droplet}

The displacement field of the liquid will be calculated when the droplet's north pole oscillates around its equilibrium position at the resonance frequency $\omega_{0}$. Between an oblate and a prolate position, $R_{p}$ varies from $R_{p_{0}}-\Delta R_{p}$ to $R_{p_{0}}+\Delta R_{p}$. A mathematical transformation that will expand the $Z$ axis and contract the $X$ and $Y$ axes is introduced to give the displacement field. The viscous dissipated power is then derived from the Navier-Stokes equations yielding

$$
\left\langle P_{v}\right\rangle=\frac{3}{2} \mu \omega_{0}^{2} V \frac{\Delta R_{p}^{2}}{R_{p_{0}}^{2}} .
$$

(The detailed calculation is in the Appendix.) Combining Eq. (6.4) and Eq. (6.3) yields the viscosity:

$$
\mu=\frac{2 \pi}{3} R_{p_{0}}^{2} \rho \Delta f_{r}
$$

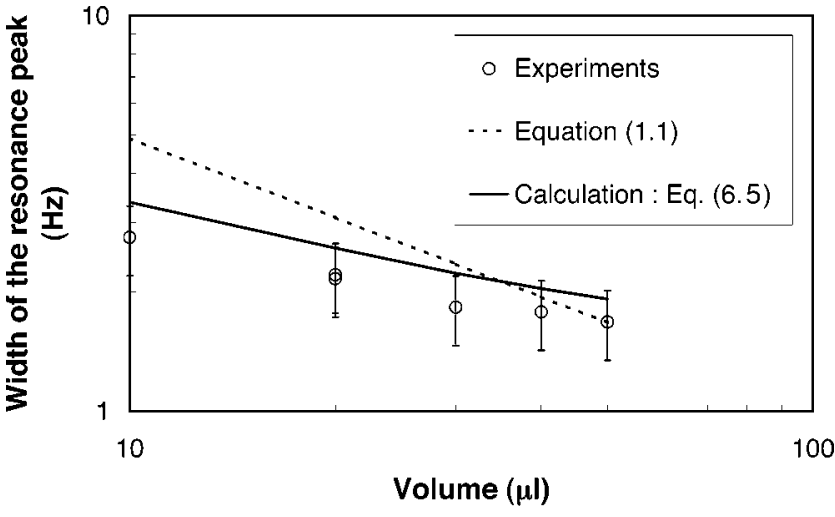

FIG. 6. Comparison of the experimental results for the width of the resonance peak with the estimation from variational approaches using ellipsoidal shapes. The solution given by Eq. (1.1) is shown for comparison $(---)$.

This equation relates the width of the resonance peak to the viscosity $\mu$ of the liquid.

For the limiting case of small oscillations around a spherical position $\left(R_{p_{0}}=R\right)$, this equation differs from Eq. (1.1) only by a coefficient $5 / 3$, which is quite reasonable considering the simplicity of our approach. Thus, as gravity (or droplet volume) increases, viscosity should be interpolated from Eq. (1.1) to Eq. (6.5).

\section{Discussion}

Figure 6 shows a good agreement between the measured and calculated $\Delta f_{r}$ as a function of the volume for a calibrated silicon oil drop, whose viscosity is $10.1 \mathrm{mPa}$. This result underlines the necessity to take into account the flattened shape of the drop. The displacement field is not the same for a spherical and an oblate oscillating drop, and thus the relation between peak width and viscosity is also different.

To validate our setup for viscosity measurements, experiments were performed on drops of water-glycerol with various fractions of glycerol (from $20 \%$ to $85 \%$ ). For each drop, the viscosity calculated by Eq. (6.5) [with the value of $R_{p_{0}}$ resulting from the minimization of the energy (see Ref. [4]) and the measurement of $\Delta f_{r}$ ] is compared with data extracted from the literature [15]. Figure 7 displays the calculated and the real viscosity versus the fraction of glycerol. The good correlation between calculated and real viscosity shows that contactless viscosity measurements can be performed with this setup with an accuracy of about $20 \%$.

\section{NONLINEAR EFFECTS}

In order to explain the distortion of the resonance peak introduced in Sec. III (see Fig. 2), let us start with a geometrical consideration: an oblate ellipsoid has its maximum total curvature along the equator whereas a prolate ellipsoid concentrates the maximum curvature at the two poles. The oblate drop stores more surface energy than the prolate one. This is confirmed by the asymmetric profile of the potential energy curve calculated in the variational approach in Sec. V.

As mentioned in Ref. [4], we stress that the energy depen- 


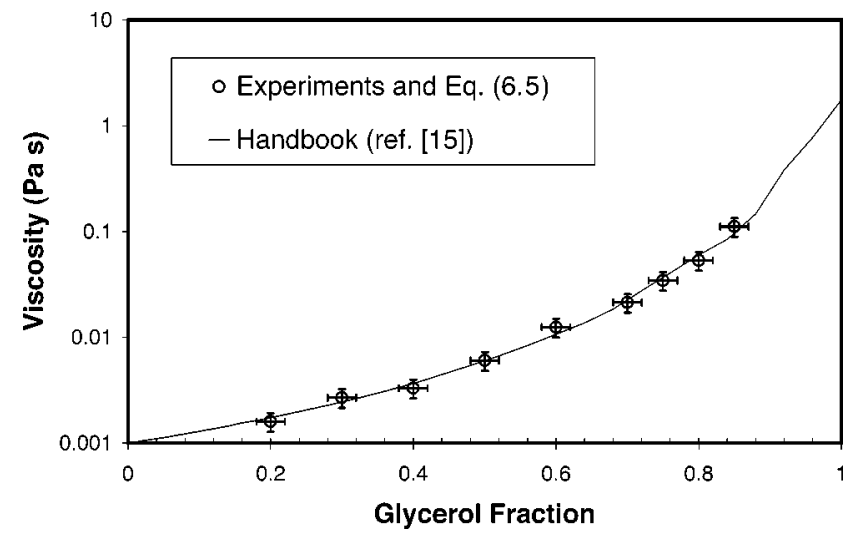

FIG. 7. Viscosity of a $50 \mu 1$ water-glycerol droplet at various fractions of glycerol, analyzed using the viscoelastic model [Eq. (6.5)] compared with data from the literature (Ref. [15]).

dence on polar-radius coordinates is not symmetrical around its minimum: this means that the drop behaves as a spring easier to expand than to compress (in terms of the polar radius). The viscoelastic model can then be modified by introducing anharmonic terms. The relation between the force $F$ and the deformation $\Delta R_{p}$ of such a spring would be

$$
F=\omega_{0}^{2} M \Delta R_{p}+\alpha M \Delta R_{p}^{2}+\beta M \Delta R_{p}^{3},
$$

$M$ being the mass of the system, $\lambda$ the dissipation coefficient, $\omega_{0}$ the resonance angular frequency, $F$ the amplitude of the excitation force, and $\alpha$ and $\beta$ the second and third order anharmonicity coefficients. The corresponding equation of motion is

$$
\ddot{R}_{p}+\dot{R}_{p} 2 \lambda+\omega_{0}^{2} R_{p}+\alpha R_{p}^{2}+\beta R_{p}^{3}=\frac{F}{M} \cos \omega t .
$$

This is the anharmonic oscillator equation treated by Landau and Lifshitz in Ref. [16]. An expansion to third order in $\epsilon=\omega-\omega_{0}$ gives the relation between the forced oscillation frequency $\omega$ and amplitude $\Delta R_{p}$ :

$$
\epsilon= \pm \sqrt{\left(\frac{F}{2 M \omega_{0} \Delta R_{p}}\right)^{2}-\lambda^{2}}+\left(\frac{3 \beta}{8 \omega_{0}}-\frac{5 \alpha^{2}}{12 \omega_{0}^{3}}\right) \Delta R_{p}^{2} .
$$

The first term is the classical harmonic oscillator equation. The nonlinear effect is given by the last term of this equation, which is negligible for small amplitude of excitation. But as $F$ (or $\Delta R_{p}$ ) increases, the peak is shifted and then distorted, leading to the appearance of hysteresis when $\Delta R_{p}(\omega)$ exhibits three values for a given $\omega$. In Fig. 8, it can be observed that when frequencies are scanned upward, the system follows the resonance curve until point $A$ (angular frequency $\left.\omega_{\text {sup }}\right)$, where it has no other possibility than to jump to point $B$. In contrast, for a downward scanning, between $B$ and $C$, the system usually stays on the upper branch up to point $C$ (angular frequency $\omega_{\text {inf }}$ ) and then falls down from $C$ to $D$. The amplitude of hysteresis is set to be the difference between the angular frequencies at $A$ and $D$ : $\omega_{\text {sup }}-\omega_{\text {inf }}$.

Since the amplitude $a$ of the excitation oscillations imposed at the south pole is much smaller than the resonance

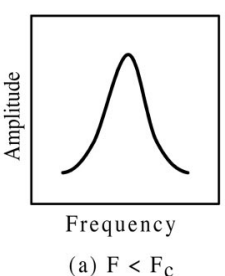

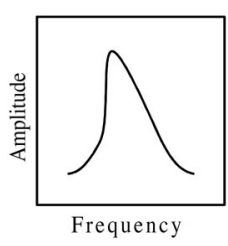

(b) $\mathrm{F}=\mathrm{F}_{\mathrm{C}}$

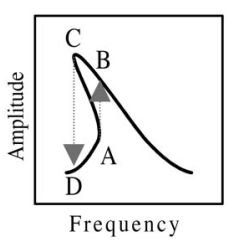

(c) $\mathrm{F}>\mathrm{F}_{\mathrm{c}}$
FIG. 8. Frequency-amplitude diagram for different excitation force $F$. With increasing excitation the peak becomes more and more distorted and finally hysteretic behavior appears.

amplitude $\Delta R_{p}$, one can simultaneously assume a nonlinear expression such as Eq. (7.1) for the response and a linear relation between the force $F$ and the amplitude $a$. The effective stiffness of the droplet is given by $M \omega_{0}^{2}$. Therefore, one can write

$$
F=M \omega_{0}^{2} a .
$$

The critical force $F_{c}$ and excitation amplitude $a_{c}$ at which this hysteresis appears are given by (Ref. [16])

$$
F_{c}^{2}=\frac{32 \omega_{0}^{2} M^{2} \lambda^{3}}{3 \sqrt{3}\left|\frac{3 \beta}{8 \omega_{0}}-\frac{5 \alpha^{2}}{12 \omega_{0}^{3}}\right|} \text { and } a_{c}^{2}=\frac{32 \lambda^{3}}{3 \sqrt{3} \omega_{0}^{2}\left|\frac{3 \beta}{8 \omega_{0}}-\frac{5 \alpha^{2}}{12 \omega_{0}^{3}}\right|} \text {. }
$$

In the experiments, the frequency $\omega$ and the amplitude $a$ are the control parameters.

The eigenfrequency $\omega_{0}$, calculated using the variational approach agrees with the measured value given by the position of the peak [4]. The coefficients $\alpha$ and $\beta$ are estimated from the anharmonicity of the energy profile. Note that the deformed resonance peak is bent toward negative frequency shifts. As mentioned in Ref. [10], this corresponds to a negative third order anharmonic coefficient $\beta$, in accordance with the energy profile. The critical amplitude above which hysteresis appears is related to the dissipation coefficient $\lambda$ $=\pi \Delta f_{r}$ through Eq. (7.5). The dissipation coefficient is related to the viscosity of the droplet through Eq. (6.5) derived from the energetic approach of Sec. VI.

Above $a_{c}$, a hysteresis defined as a frequency band with three possible solutions (see Fig. 8) can be observed. A rough estimate for the hysteresis in frequency can be given

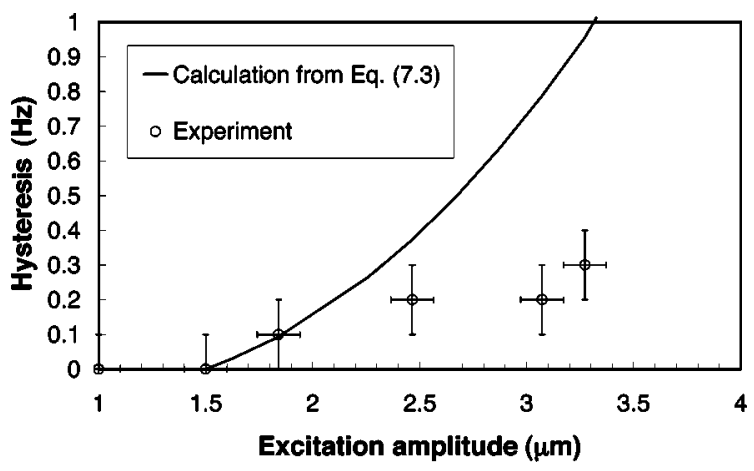

FIG. 9. Hysteresis in the frequency-amplitude diagram as a function of the excitation amplitude: comparison between experiments and theoretical values. 


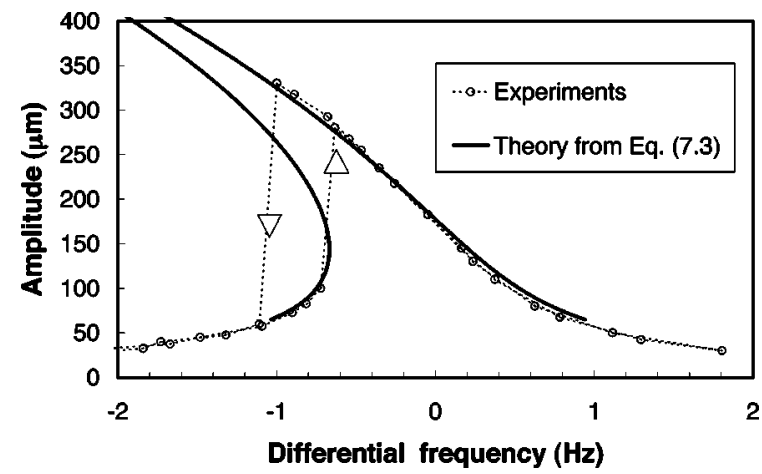

FIG. 10. Distorted resonance peak: comparison between experiments and Eq. (7.3).

by the distance between $[C D]$ and $[B A]$ on Fig. 8. In Fig. 9, the experimental hysteresis is plotted versus the excitation amplitude. For relatively low amplitudes, the estimate is in good agreement with experiments. But for amplitudes larger than $2.5 \mu \mathrm{m}$, the hysteresis is less important than predicted by this simple model. In Fig. 10, the experimental hysteresis curve is compared with the theoretical one given by Eq. (7.3). The agreement is strikingly good. However, the jump from the upper branch to the lower branch, when scanning downward in frequency, occurs well before the ultimate limit of the three valued domain. This is the origin of the discrepancy exhibited in Fig. 9.

The dependence on viscosity $\mu$ of the critical amplitude $a_{c}$ above which hysteresis occurs (or equivalently of the critical excitation force $F_{c}$ ) is shown in Fig. 11. For low viscosity droplets (less than $5 \mathrm{mPa} \mathrm{s}$ ), the experimental critical amplitude is very accurately described by Eq. (7.5). For viscosities larger than $5 \mathrm{mPa}$ s, hysteresis is observed only at much larger excitation amplitude. This transition also corresponds to an overall instability of the droplet position: the drop starts to bounce like a ball. Qualitatively, this can be understood as follows: when the excitation amplitude $a$ reaches a value $a^{*}$ (of the order $4 \mu \mathrm{m}$, Fig. 11) a new composite mode is excited. For viscosities larger than $\sim 5 \mathrm{mPa}$ s, this will occur before reaching the threshold $a_{c}$ for the "pure" deformation mode $\left[a_{c} \propto \lambda^{3 / 2}\right.$, Eq. (7.5) or the solid curve in Fig. 11]. In our view, this new mode comprises two coupled components: deformation and bouncing.

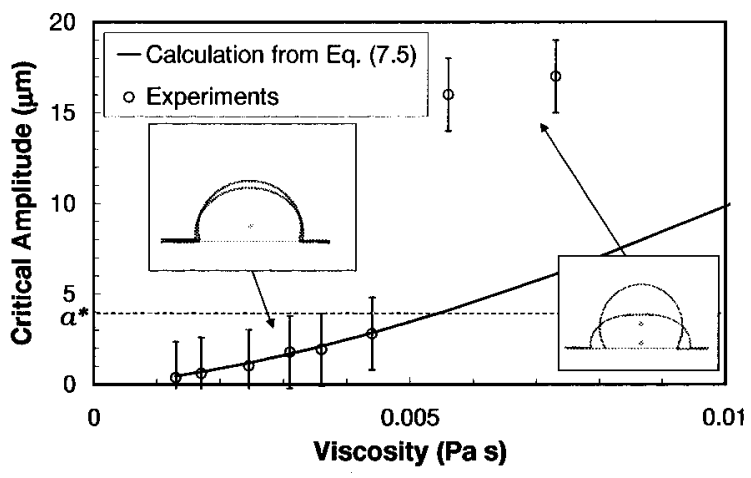

FIG. 11. The viscosity dependence of the critical excitation amplitude: comparison between theory from Eq. (7.5) and experiments. Dashed line stands for $a^{*}$, where new composite mode (bouncing + deformation) sets in.

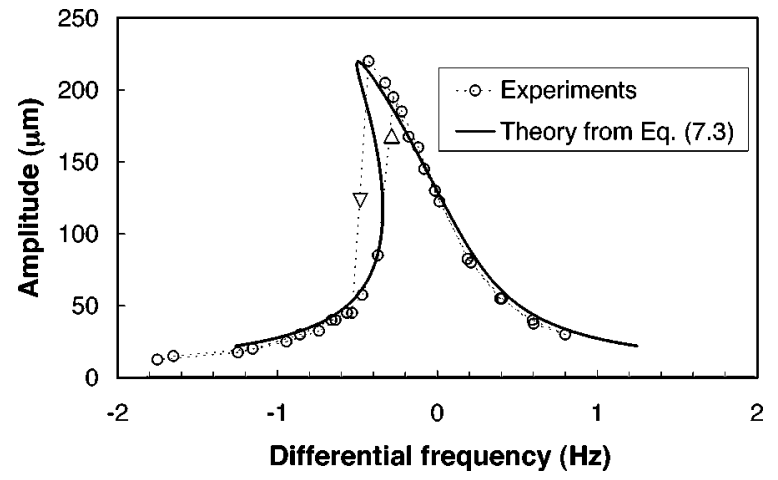

FIG. 12. An example of distorted resonance peak close to the critical excitation amplitude: comparison between experiments and theory from Eq. (7.3).

This is likely to be describable, again, in terms of anharmonic oscillations, but with different dynamical parameters $\left(M, \omega_{0}, \alpha, \beta\right.$, etc.). If we consider that the main change is in $\omega_{0}$, then Eq. (7.5) indicates that $a_{c}$ increases when $\omega_{0}$ decreases, in agreement with Fig. 11.

Finally, Fig. 12 shows a comparison between experimental and theoretical hysteresis curves just above the critical amplitude $a_{c}$. It shows that the present theory with no adjustable parameters describes correctly both the harmonic and the anharmonic effects.

\section{CONCLUSION}

The main difference between the gas-film-levitation experimental conditions and the theory of free oscillation presented in the Introduction is the experimentally observed nonspherical shape of the droplet at equilibrium. An Archimedean arc can accurately represent the real shape, and leads to results similar to an ellipsoid of revolution concerning the resonance frequency. Thus, an ellipsoidal approximation can be used to model the dynamic of the droplet.

A relation between the viscosity and the width of the measured resonance peak has been derived. It takes into account both shape and boundary conditions and can lead to accurate contactless measurement of dynamic viscosity of liquids from $2 \mathrm{mPa}$ s to $150 \mathrm{mPa}$.

The asymmetric profile of the energy curves points to the nonlinear character of the oscillations due to anharmonicity. A detailed description of the nonlinearity has been proposed to explain quantitatively the distortion of the peak for high amplitudes and the occurrence of hysteretic behavior.

The experimental setup described and validated in this paper can be a tool to measure surface tension and viscosity accurately. Contactless viscosity measurements could be useful to study the rheology of materials in the semisolid state without perturbing the dynamics of crystallization (no container). Suspensions could also be studied without the classical boundary layer problems that frequently appear in Couette rheometry (no container and therefore no heterogeneous nucleation; for a recent preliminary report, see Ref. [17]).

\section{ACKNOWLEDGMENTS}

The authors want to thank the DEM/SPCM/LPSI from CEA Grenoble for providing the experimental facility. One 
of the authors (M. Perez) was supported by a MENRT grant, and partial financial support from Région Rhône-Alpes is gratefully acknowledged.

\section{APPENDIX: CALCULATION OF THE VISCOUS DISSIPATED POWER}

The viscous dissipated power is calculated by writing the velocity field inside the droplet, which itself is evaluated from the displacement field.

If $M_{p}\left(x_{p}, y_{p}, z_{p}\right)$ is a point of the prolate shape, $M_{o}\left(x_{o}, y_{o}, z_{o}\right)$, a point of the oblate shape, and $M(x, y, z)$, a point of the equilibrium shape, to ensure volume conservation, we have

$$
\begin{gathered}
x_{p}=x_{o} A^{-1 / 2}, \\
y_{p}=y_{o} A^{-1 / 2} \text { with } A=\frac{R_{p_{0}}+\Delta R_{p}}{R_{p_{0}}-\Delta R_{p}}, \\
z_{p}=z_{o} A
\end{gathered}
$$

The displacement field resulting from the oscillation between the prolate and the oblate position is

$$
\begin{gathered}
x(t)=\frac{x_{p}+x_{o}}{2}+\frac{x_{p}-x_{o}}{2} \sin (\omega t)=x+x \sin (\omega t) \frac{1-\sqrt{A}}{1+\sqrt{A}}, \\
y(t)=\frac{y_{p}+y_{o}}{2}+\frac{y_{p}-y_{o}}{2} \sin (\omega t)=y+y \sin (\omega t) \frac{1-\sqrt{A}}{1+\sqrt{A}}, \\
z(t)=\frac{z_{p}+z_{o}}{2}+\frac{z_{p}-z_{o}}{2} \sin (\omega t)=z+z \sin (\omega t) \frac{A-1}{A+1}
\end{gathered}
$$

[1] J. Granier and C. Potar (unpublished).

[2] A. Janke and G.H. Frischat, Glass Sci. Technol. (Amsterdam) 71, 855 (1990)

[3] C. Parayre et al., CEA-DTA Int. Report DEM N. DR/17/96, 1997 (unpublished).

[4] M. Perez, Y. Bréchet, L. Salvo, M. Papoular, and M. Suéry, Europhys. Lett. 47, 189 (1999).

[5] J.C. Barbé, C. Parayre, M. Daniel, M. Papoular, and N. Kernevez, Int. J. Thermophys. 20, 1071 (1999).

[6] E. Gorges and I. Egry, J. Mater. Sci. 30, 2517 (1995).

[7] I. Egry, G. Lohofer, and G. Jacobs, Phys. Rev. Lett. 75, 4043 (1995).

[8] I. Egry, G. Lohofer, I. Seyhan, S. Schneider, and B. Feuerbacher, Appl. Phys. Lett. 73, 462 (1998).

[9] K.C. Mills and R.F. Brooks, Mater. Sci. Eng., A 178, 77 (1994)
The velocity field is the first derivative of the displacement field:

$$
\begin{aligned}
& v_{x}(t)=x \omega \cos (\omega t) \frac{1-\sqrt{A}}{1+\sqrt{A}}, \\
& v_{y}(t)=y \omega \cos (\omega t) \frac{1-\sqrt{A}}{1+\sqrt{A}}, \\
& v_{z}(t)=z \omega \cos (\omega t) \frac{A-1}{A+1} .
\end{aligned}
$$

The divergence of the velocity field is not strictly zero as expected for a noncompressible fluid, but is negligible compared to any of its components. The viscous dissipated power can be derived from the Navier-Stokes equations (see, for instance, Ref. [12]):

$$
\left\langle P_{v}\right\rangle=\frac{\omega_{0}}{2 \pi} \int_{0}^{2 \pi / \omega_{0}} \frac{\mu}{2} \int_{V}\left(\frac{\partial v_{i}}{\partial x_{j}}+\frac{\partial v_{j}}{\partial x_{i}}\right)^{2} d V d t,
$$

giving

$$
\left\langle P_{v}\right\rangle=\frac{\omega_{0}}{2 \pi} \int_{0}^{2 \pi / \omega_{0}} \frac{\mu}{2} V\left[\left(2 \frac{\partial v_{x}}{\partial x}\right)^{2}+\left(2 \frac{\partial v_{y}}{\partial y}\right)^{2}+\left(2 \frac{\partial v_{z}}{\partial z}\right)^{2}\right] d t
$$

For small oscillations $\left(\Delta R_{p} / R_{p_{0}}<<1\right)$, a first order power expansion in $\Delta R_{p} / R_{p_{0}}$ gives for the viscous dissipated power

$$
\left\langle P_{v}\right\rangle=\frac{3}{2} \mu \omega_{0}^{2} V \frac{\Delta R_{p}^{2}}{R_{p_{0}^{2}}}
$$

[10] M. Papoular and C. Parayre, Phys. Rev. Lett. 78, 2120 (1997).

[11] H. Lamb, Hydrodynamics (Cambridge University Press, Cambridge, 1932).

[12] L. Landau, and E. Lifshitz, Fluid Mechanics, Course of Theoretical Physics Vol. 6 (Pergamon Press, Oxford, 1959).

[13] D.L. Cumming and D.A. Blackburn, J. Fluid Mech. 224, 395 (1991).

[14] N. Coutris and O. Gentil, CEA-DTE Int. Report SETh/LEF/ 89.29, 1989 (unpublished).

[15] Handbook of Chemistry and Physics, 62nd ed. (CRC Press, Boca Raton, FL, 1981).

[16] L. Landau, and E. Lifshitz, Mechanics, Course of Theoretical Physics (Pergamon Press, Oxford, 1959).

[17] J.C. Barbé, M. Perez, and M. Papoular, J. Phys.: Condensed Matter (to be published). 